\title{
Inhibition of hypoxia-induced angiogenesis by sodium butyrate, a histone deacetylase inhibitor, through hypoxia-inducible factor-1 $\alpha$ suppression
}

\author{
SE-HEE KIM ${ }^{1}$, KYU-WON KIM ${ }^{1}$ and JOO-WON JEONG ${ }^{2}$ \\ ${ }^{1}$ Research Institute of Pharmaceutical Sciences, College of Pharmacy, Seoul National University, \\ Seoul 151-742; ${ }^{2}$ Department of Anatomy and Neurobiology, Biomedical Science Institute, \\ School of Medicine, Kyung Hee University, Seoul 130-701, Korea
}

Received December 1, 2006; Accepted January 24, 2007

\begin{abstract}
Hypoxia-inducible factor-1 (HIF-1) plays a pivotal role in cellular response to low oxygen concentration, such as angiogenesis in tumors. Here, we found that a histone deacetylase inhibitor, sodium butyrate, inhibits the hypoxiainduced induction and activity of HIF- $1 \alpha$ in HT1080 human fibrosarcoma cells. Moreover, sodium butyrate also suppressed the hypoxia-stimulated angiogenic effects and downregulated HIF- $1 \alpha$ and vascular endothelial growth factor expression in vascular endothelial cells. These findings suggest that sodium butyrate may play important roles in tumor suppression via inhibition of HIF-1 $\alpha$ mediated angiogenesis under hypoxic conditions.
\end{abstract}

\section{Introduction}

Hypoxia detected in the central region of solid tumors can be a leading cause of angiogenesis, a fundamental determinant of malignant tumor progression, by activating the expression of angiogenic factors $(1,2)$. A key factor in this process is hypoxia-inducible factor (HIF), which regulates transcription of hypoxia-activated genes and consists of HIF- $1 \alpha$ and HIF-1ß heterodimers. The $\alpha$ subunit of HIF-1 is rapidly degraded under normoxic conditions and stabilized under hypoxia, while HIF-1ß is expressed constitutively (3).

Histone deacetylases (HDACs) are enzymatic components of multi-protein complexes that are recruited by transcription factors to specific DNA regulatory sequences. Major functions of HDACs are to control gene expression, transport ubiquitinated protein aggregates, and deacetylate proteins (4-6). Global suppression of HDAC activity by chemical inhibitors generally raises cell cycle arrest and may affect cell proliferation and

Correspondence to: Dr J.-W. Jeong, Department of Anatomy and Neurobiology, School of Medicine, Kyung Hee University, Seoul 130-701, Korea

E-mail: jjeong@khu.ac.kr

Key words: sodium butyrate, angiogenesis, hypoxia-inducible factor- $1 \alpha$, vascular endothelial growth factor differentiation $(7,8)$. Specific HDAC inhibitors have been used to explain HDAC function and suggested as a therapy for some types of cancers (9). Consequently, they have been shown to have a potent anti-tumor activity in human xenograft animal models and are being actively tested in clinical trials.

Sodium butyrate, a short-chain fatty acid naturally present in the colon, is an HDAC inhibitor and is able to modulate a variety of fundamental cellular processes and to induce cell cycle arrest, differentiation and apoptosis in transformed cells (10-13). Currently, sodium butyrate is being evaluated as an anti-neoplastic therapeutic (14), and clinical trials of butyrate and its derivatives in cancer patients have already been initiated $(15,16)$.

In the present study, we discovered that sodium butyrate decreases HIF- $1 \alpha$ protein level, transactivation function of HIF-1, and vascular endothelial growth factor (VEGF) expression in hypoxic conditions. In addition, sodium butyrate exhibited an inhibitory effect by in vitro angiogenesis assay, migration and tube formation of endothelial cells. Moreover, sodium butyrate was able to repress angiogenesis in embryo in vivo. These results demonstrate that sodium butyrate inhibits hypoxia-induced angiogenesis by downregulating HIF-1 activity including HIF-1 $\alpha$ stability.

\section{Materials and methods}

Reagents and antibodies. Sodium butyrate was purchased from Sigma (St. Louis, MO). Anti-HIF- $1 \alpha$ and VEGF antibodies were purchased from BD Pharmigen (San Diego, CA) and Santa Cruz Biotechnology (Santa Cruz, CA), respectively.

Cell culture and hypoxic condition. Human embryonic kidney (HEK) 293 and human fibrosarcoma HT1080 cells were maintained in Dulbecco's modified Eagle's medium (DMEM) (Invitrogen, Grant Island, NY) supplemented with $10 \%$ fetal bovine serum (FBS) (Invitrogen) and 1\% antibiotics (Invitrogen). Human umbilical vein endothelial cells (HUVECs) were grown in Medium 199 (Invitrogen) supplemented with $20 \% \mathrm{FBS}, 100$ units/ml penicillin, $100 \mathrm{~g} / \mathrm{ml}$ streptomycin, $3 \mathrm{ng} / \mathrm{ml}$ basic fibroblast growth factor (Upstate Biotechnology, New York, NY), and 5 units/ml heparin (Sigma). For hypoxic conditions, cells were incubated in $5 \% \mathrm{CO}_{2}$ with $1 \% \mathrm{O}_{2}$ 
balanced with $\mathrm{N}_{2}$ in a hypoxic chamber (Forma Scientific, Marietta, $\mathrm{OH})$.

Western blot analysis. Proteins were separated by SDS-PAGE and transferred to a nitrocellulose membrane (Amersham Pharmacia Biotech, Arlington Heights, IL). The membrane was blocked with $5 \%$ skim milk in phosphate-buffered saline containing $0.1 \%$ Tween-20 for $1 \mathrm{~h}$ at room temperature, incubated with the primary antibody at room temperature for $1 \mathrm{~h}$ or at $4^{\circ} \mathrm{C}$ overnight, followed by incubation with horseradish-conjugated antibody against rabbit or mouse immunoglobulins at room temperature for $50 \mathrm{~min}$, and was then developed by the ECL plus Western detection system (Amersham Pharmacia Biotech).

Reverse transcription-polymerase chain reaction (RT-PCR). Total RNA was isolated from cell cultures by a single-step procedure with TRI reagent (Life Technologies, Gaithersburg, MD). cDNA was synthesized from $5 \mu \mathrm{g}$ of total RNA with 200 units of MMLV-RT (Promega, Madison, WI) and 500 ng of oligo-dT primer. PCR reaction was performed on the first strand cDNAs using the PCR reaction kit (Takara, Japan) and primer sets. Amplification by PCR was performed using an automated thermal cycler (Perkin-Elmer). Oligonucleotide primers for PCR were designed as follows: $\beta$-actin, 5'-GAC TACCTCATGAAGATC-3' and 5'-GATCCACATCTGCT GGAA-3'; HIF-1 $\alpha, 5$ '-CAGAAGATACAAGTAGCCTC-3' and 5'-CTGCTGGAATACTGTAACTG-3'; VEGF, 5'-GAG AATTCGGCCTCCGAAACCATGAACTTTCTGT-3' and 5'-GAGCATGCCCTCCTGCCCGGCTCACCGC-3' .

Transient transfection and luciferase assay. Plasmids $(5 \mu \mathrm{g})$ were transfected to $5 \times 10^{5}$ HEK293 cells/60-mm dish, with proper recombination of effector plasmids, pSV40promoterEpoHRE-Luc and control plasmid pCMV-ß-gal and pBOShHIF- $1 \alpha$ and pBOS-hARNT using Lipofectamine reagent according to the instructions of the manufacturer (Invitrogen). These vectors were kindly provided by Dr Fujii Kuriyama (Tohoku University, Japan) (17). After transfection, cells were harvested and extracts were prepared using reporter lysis buffer (Promega). Cell lysates were analyzed for luciferase activity using an assay kit (Promega) and luminometer (Turner Design, Sunnyvale, CA). Extracts were also assayed for $\beta$ galactosidase enzyme and protein concentration with the protein assay kit (Sigma). Each extract was assayed three times, and the mean RLU was corrected by values obtained from an extract prepared from empty vector-transfected cells. The relative luciferase activity was calculated as RLU/Bgalactosidase.

Tube formation assay. Matrigel ( $250 \mu \mathrm{l} ; 10 \mathrm{mg} / \mathrm{ml}$ ) (Becton Dickinson, Franklin Lakes, NJ) was pipetted onto 24-well culture plates and polymerized for $30 \mathrm{~min}$ at $37^{\circ} \mathrm{C}$. HUVECs $\left(1 \times 10^{5}\right.$ cells) were seeded on the surface of Matrigel. Then sodium butyrate was added and incubated for $24 \mathrm{~h}$ under hypoxic conditions. Morphologic change of cells was observed under a microscope and photographed at x40 magnification.

Wounding migration assay. Wounding migration assay was performed as previously described (18). HUVECs were plated

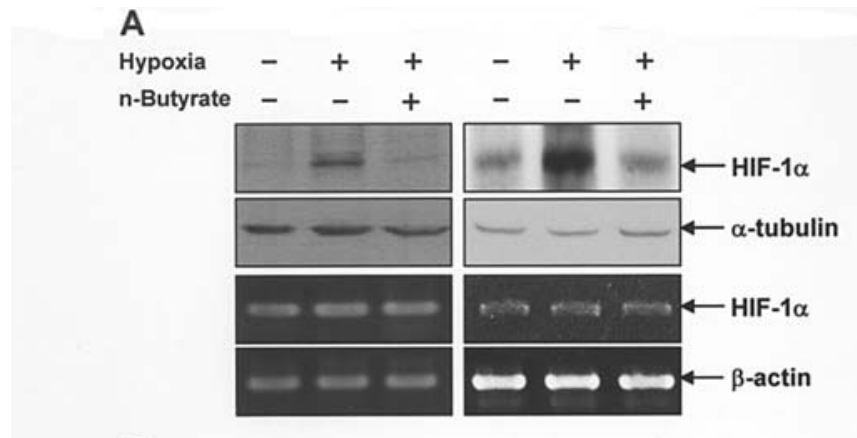

B
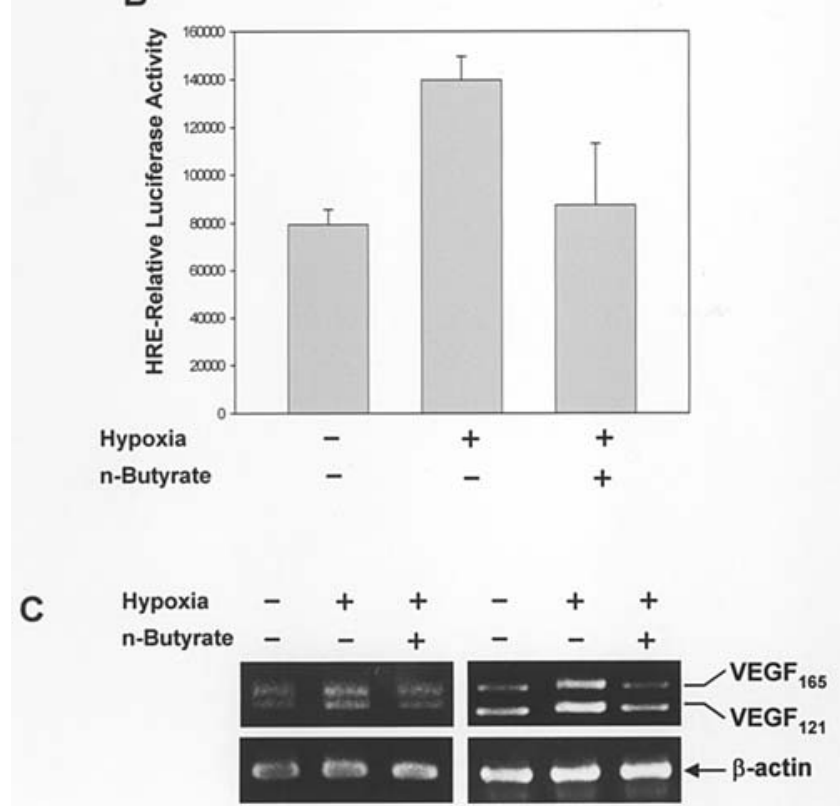

Figure 1. Sodium butyrate decreases HIF-1 activities. (A) Cells were incubated with or without $1 \mathrm{mM}$ sodium butyrate (n-butyrate) at the indicated concentrations under normoxia or hypoxia for $16 \mathrm{~h}$. Western blotting (top two) and RT-PCR (bottom two) analyses against HIF-1 $\alpha$ were performed on total protein and RNA isolated from HEK293 (left) and HT1080 (right) cells treated as indicated. $\alpha$-tubulin and $\beta$-actin served as loading controls. (B) HEK293 cells were cotransfected with pSV40pro-EpoHRE-Luc $(1 \mu \mathrm{g})$, pBOS-hHIF-1 $\alpha(0.1 \mu \mathrm{g})$, and pBOS-hARNT $(0.1 \mu \mathrm{g})$. Transfected cells were incubated for an additional $16 \mathrm{~h}$ with or without $1 \mathrm{mM}$ sodium butyrate as indicated. The mean and standard deviation based on three independent transfections are shown. (C) HEK293 (left) and HT1080 (right) cells were exposed to hypoxia for $16 \mathrm{~h}$ in the absence or presence of the sodium butyrate $(1 \mathrm{mM})$. RT-PCR analysis was performed using specific primer for VEGF and $ß$-actin.

onto $0.3 \%$ gelatin-coated $60-\mathrm{mm}$ culture dishes. At $90 \%$ confluence, the endothelial monolayers were marked with an injury line and wounded $2 \mathrm{~mm}$ in width with a sterile razor blade. Plates were rinsed with serum-free medium to remove cellular debris. Fresh medium with sodium butyrate and $1 \mathrm{mM}$ thymidine were then added. HUVECs were allowed to migrate for $24 \mathrm{~h}$ and rinsed with serum-free medium, and photographs were taken through an inverted microscope (x40). The number of cells that moved beyond the reference line was counted. The experiment was performed more than three times.

Chemoinvasion assay. Chemoinvasion assay was carried out by the method of Saiki et al (19). The lower and upper sides of $8 \mathrm{~m}$ porosity polycarbonate filters were coated with $0.5 \mathrm{mg} / \mathrm{ml}$ type I collagen and $0.5 \mathrm{mg} / \mathrm{ml}$ Matrigel, respectively. The 
lower compartment contained sodium butyrate-treated media containing $0.1 \mathrm{mg} / \mathrm{ml}$ bovine serum albumin and HUVECs placed in the upper part of Transwell plate. Cell invasion was determined by counting whole cell numbers at single filter with optical microscopy at $\mathrm{x} 40$ magnification. The experiment was assayed more than three times with duplicate independent samples.

Chorioallantoic membrane (CAM) assay. Thermanox coverslips (Invitrogen) were loaded with $5 \mu 1$ of concentrated (50X) conditioned media. After air-drying, loaded coverslips were then applied to the CAM surface of 4-day-old chick embryos. After two days, an appropriate volume of $10 \%$ fat emulsion (10\% Intralipose) was injected into the 6-day-old embryo chorioallantois, and angiogenesis was observed under a microscope.

\section{Results}

Sodium butyrate downregulates the activity of HIF-1. To investigate the effect of sodium butyrate on the expression of HIF-1 $\alpha$, we treated HEK293 and HT1080 cells with sodium butyrate for $16 \mathrm{~h}$ under hypoxic conditions. As shown in Fig. 1A, sodium butyrate downregulated the protein level of HIF-1 $\alpha$ stabilized by hypoxia, whereas it did not affect the mRNA level of HIF-1 $\alpha$. This suggests that the stability of the HIF- $1 \alpha$ protein level is reduced by the treatment of sodium butyrate. To check whether the reduced HIF-1 $\alpha$ protein by sodium butyrate is involved in the HIF-1 transcriptional activity, we used a luciferase reporter system, pSV40promoterEpoHRE-Luc reporters. Upon transient transfection of HIF-1 $\alpha$, ARNT, and EpoHRE-Luc vectors, reporter activity was markedly increased in cells exposed to hypoxia compared with normoxia (Fig. 1B). However, the treatment of sodium butyrate was shown to decrease the transcriptional activity of HIF-1 under hypoxic conditions (Fig. 1B). Furthermore, to evaluate whether sodium butyrate modulates the hypoxiaresponse element (HRE)-containing VEGF gene expression level, HEK293 and HT1080 cells were treated with sodium butyrate for $16 \mathrm{~h}$ under hypoxia. As shown in Fig. 1C, the increased expression of VEGF mRNA by hypoxia was downregulated in the case of treatment with sodium butyrate. These results suggest that sodium butyrate negatively regulates the hypoxia-inducible activity of HIF-1.

Inhibition of in vivo and in vitro angiogenesis by sodium butyrate. The antiangiogenic effect of sodium butyrate was investigated in vivo, using a CAM inhibition assay. As shown in Fig. 2A, sodium butyrate produced a significant decrease in the development of angiogenesis in a chick embryo without any sign of thrombosis and hemorrhage compared with control. To investigate the direct effect of sodium butyrate on the vascular endothelial cells under hypoxic conditions, in vitro angiogenesis assays were conducted using human endothelial cells, HUVECs. Since it has been demonstrated that the hypoxic conditions stimulate vessel network formation of cultured endothelial cells $(20,21)$, we used hypoxia for the induction of angiogenesis. HUVECs that were seeded on Matrigel beds $(10 \mathrm{mg} / \mathrm{ml})$ were treated with sodium butyrate, and incubated for $24 \mathrm{~h}$ under hypoxic conditions. As shown in Fig. 2B, HUVECs formed normally hollow tubes under normoxia, whereas capillary network formation was further stimulated under hypoxia, resulting in elongated and robust tube-like structures. In this condition, sodium butyrate strongly inhibited the hypoxia-stimulated network by much foreshortened and severely broken tubes (Fig. 2B). These results demonstrated that sodium butyrate potently inhibits angiogenesis in vivo and in vitro.

Sodium butyrate decreases the hypoxia-induced angiogenic effects of HUVECs. To investigate the effect of sodium butyrate on endothelial cell migrating activity, we performed the wounding migration assay using HUVECs. Fig. 3A showed that the mobility of HUVECs was significantly enhanced under hypoxic conditions similar to a previous report $(20,21)$, whereas the migratory ability of the cells was dramatically reduced by treatment of sodium butyrate compared with hypoxia alone. As an important property of angiogenesis, migrating endothelial cells must break their basement membrane and transverse through it to form new blood vessels and hypoxia can stimulate endothelial cell invasion. Thus, the effect of sodium butyrate on the vascular endothelial cell invasion was studied using a polycarbonate filter of transwell coated with the Matrigel. The HUVECs were placed in the filter and allowed to invade under normoxic or hypoxic conditions. As shown in Fig. 3B, sodium butyrate significantly inhibited the hypoxia-stimulated invasiveness of the HUVECs.

Loss of HIF-1 $\alpha$ in endothelial cells inhibits a number of important parameters of endothelial cell behavior during angiogenesis, resulting from the decreased level of hypoxiadriven VEGF expression (21). We therefore examined whether sodium butyrate could influence the expression patterns of HIF- $1 \alpha$ and VEGF in hypoxic endothelial cells. As expected, sodium butyrate suppressed HIF-1 $\alpha$ and VEGF protein levels under hypoxia in endothelial cells (Fig. 3C). These results suggest that the downregulated HIF-1 $\alpha$ and VEGF by sodium butyrate could inhibit the hypoxia-stimulated migration, invasion, and tubular formation of endothelial cells.

\section{Discussion}

Recently, HDAC inhibitors such as trichostatin A (TSA), LAQ824, FK228, and sodium butyrate have been reported to downregulate HIF-1 $\alpha$ stability in cancer cell lines $(3,18,22,23)$. This has suggested some additional mechanisms underlying HIF- $1 \alpha$ inhibition by HDAC inhibitors. Kong et al reported that TSA involves the enhanced interaction of HIF-1 $\alpha$ with HSP70 and is secondary to a disruption of the HSP70/HSP90 axis function that appears mediated by the activity of HDAC6 in human VHL-deficient cell lines (24).

However, Yoo et al suggested that HDAC1 recruited by metastasis-associated protein 1 (MTA1) deacetylates HIF-1 $\alpha$ in human cells (25). Although wild-type HIF-1 $\alpha$ is increased by MTA1 expression but decreased by TSA treatment, the expression of mutant-type HIF-1 $\alpha$ (K532R) is not altered under these conditions. This implies that TSA induces HIF- $1 \alpha$ degradation via promoting its acetylation at lysine residue. In addition, Qian et al also suggested that HIF-1 $\alpha$ inhibition by LAQ824 is associated with HIF- $1 \alpha$ acetylation and polyubiquitination (22). Based on these observations, 
A

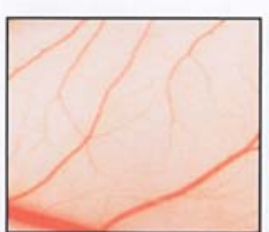

Control

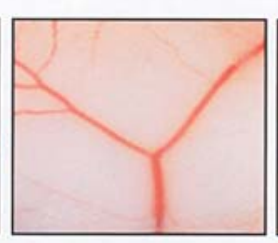

RA

B

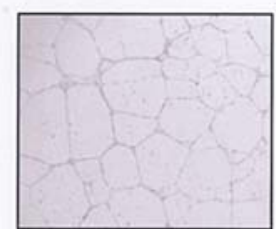

Normoxia

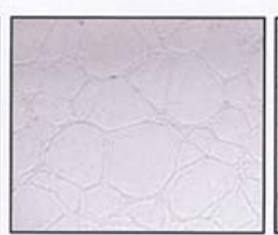

Hypoxia

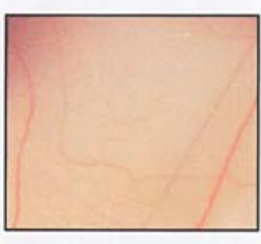

n-Butyrate

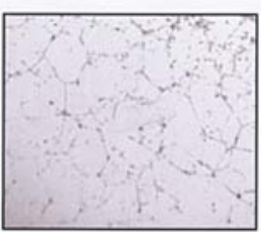

Hypoxia

+ n-Butyrate
Figure 2. Sodium butyrate suppresses angiogenesis. (A) Sodium butyrate (1 $\mathrm{mM}$ ) was treated on the CAM surface of 4-day-old chick embryos. Two days later, angiogenesis was observed under a microscope. Retinoic acid (RA) $(1 \mu \mathrm{g})$ was used as a positive control. (B) HUVECs were incubated on the Matrigel with or without sodium butyrate $(1 \mathrm{mM})$ under normoxia or hypoxia for $24 \mathrm{~h}$. The morphology of HUVECs was photographed under a microscope.
HDAC activity may be critically involved in HIF-1 $1 \alpha$ regulatory mechanism, although these molecular mechanisms require further investigation.

In the present study, we investigated whether angiogenesis of cancer under hypoxia can be regulated by sodium butyrate. The downregulation of HIF- $1 \alpha$ expression was confirmed (Fig. 1A), indicating that HDACs are involved in oxygendependent gene expression and hypoxia-induced angiogenesis. We revealed the inhibitory effect of sodium butyrate on HIF-1 activity and VEGF induction (Fig. 1B and C). This significant inhibition of VEGF by sodium butyrate under hypoxia may play a direct and critical role in the inhibition of angiogenesis. Therefore, we examined the effects of sodium butyrate on angiogenesis and revealed that sodium butyrate has a powerful potential to inhibit angiogenesis in vivo and in vitro (Fig. 2). We also found that sodium butyrate directly suppresses migratory and invasive capacities in hypoxia-stimulated endothelial cells (Fig. 3A and B). The inhibitory effects of sodium butyrate are accompanied with decreased HIF-1 $\alpha$ and VEGF expression levels in human vascular endothelial cells (Fig. 3C).

A recent report shows that the loss of hypoxia-responsive HIF- $1 \alpha$ in endothelial cells results in impaired angiogenesis. This impairment directly results in the disruption of an
A

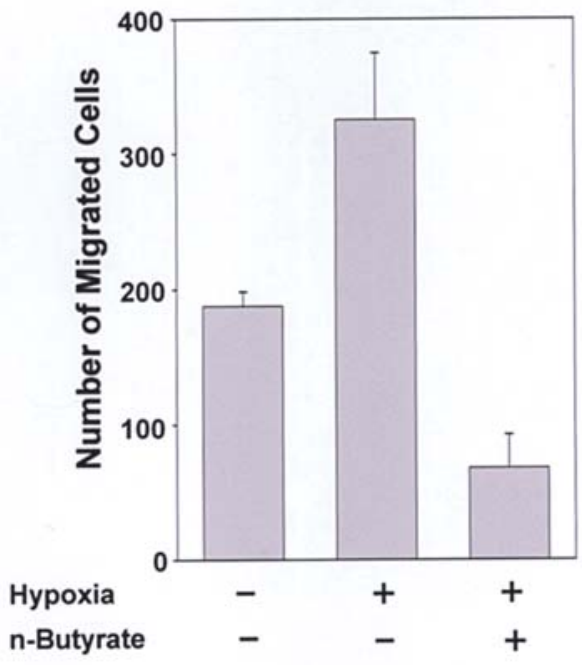

C

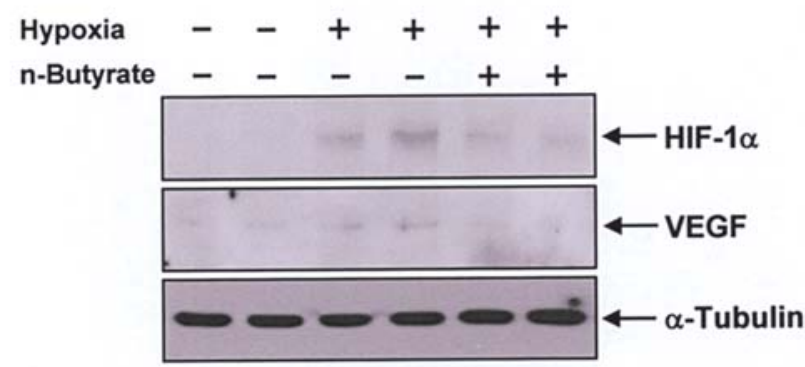

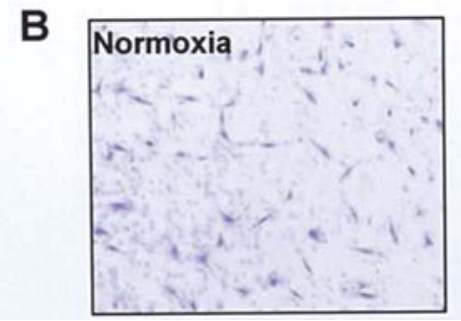
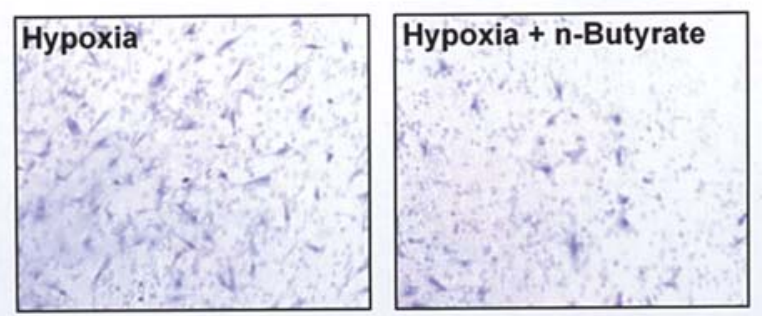

Figure 3. Sodium butyrate decreases the hypoxia-induced angiogenesis on HUVECs. (A) HUVECs were seeded on the gelatin-coated culture dish. At $90 \%$ confluence, the monolayers were wounded with a razor blade. Wounded cells were incubated with or without sodium butyrate (1 mM) under hypoxia for $24 \mathrm{~h}$. The number of HUVECs that moved beyond the reference line was counted. The data are expressed from three different experiments with duplicates. (B) HUVECs exposed to hypoxia in the absence or presence of sodium butyrate $(1 \mathrm{mM})$ were incubated on Matrigel-coated transwell chambers for $24 \mathrm{~h}$. The numbers of invaded cells were counted. (C) HUVECs were incubated under hypoxia for $16 \mathrm{~h}$ with or without $1 \mathrm{mM}$ of sodium butyrate. Total proteins were analyzed by Western blotting using anti-HIF-1 $\alpha$ and VEGF antibodies. $\alpha$-tubulin served as loading controls. 
autocrine loop, acting through HIF-1 $\alpha$ regulation of hypoxiainduced VEGF expression (21). Therefore, we figured out that the functional HIF- $1 \alpha$ and its target, VEGF, play critical roles in endothelial cells as well as tumor cells in the process of hypoxia-driven tumor angiogenesis.

We conclude that sodium butyrate downregulates HIF-1 $\alpha$ protein levels and activity, and leads to the inhibition of VEGF gene expression. Furthermore, sodium butyrate effectively blocks hypoxia-stimulated angiogenesis of vascular endothelial cells.

\section{Acknowledgements}

This work was supported by the Korea Research Foundation Grant funded by the Korean Government (MOEHRD, KRF2006-531-C00044) (to J.-W. Jeong) and the Creative Research Initiatives (NeuroVascular Coordination Research Center) of MOST/KOSEF (to K.-W. Kim).

\section{References}

1. Bergers $\mathrm{G}$ and Benjamin LE: Tumorigenesis and the angiogenic switch. Nat Rev Cancer 3: 401-410, 2003.

2. Vaupel P: The role of hypoxia-induced factors in tumor progression. Oncologist 9: 10-17, 2004.

3. Jeong JW, Bae MK, Ahn MY, Kim SH, Sohn TK, Bae MH, Yoo MA, Song EJ, Lee KJ and Kim KW: Regulation and destabilization of HIF-1alpha by ARD1-mediated acetylation. Cell 111: 709-720, 2002

4. Kawaguchi Y, Kovacs JJ, McLaurin A, Vance JM, Ito A and Yao TP: The deacetylase HDAC6 regulates aggresome formation and cell viability in response to misfolded protein stress. Cell 115: 727-738, 2003.

5. Hubbert C, Guardiola A, Shao R, Kawaguchi Y, Ito A, Nixon A, Yoshida M, Wang XF and Yao TP: HDAC6 is a microtubuleassociated deacetylase. Nature 417: 455-458, 2002.

6. Matsuyama A, Shimazu T, Sumida Y, Saito A, Yoshimatsu Y, Seigneurin-Berny D, Osada H, Komatsu Y, Nishino N, Khochbin S, Horinouchi S and Yoshida M: In vivo destabilization of dynamic microtubules by HDAC6-mediated deacetylation. EMBO J 21: 6820-6831, 2002.

7. Kouraklis G and Theocharis S: Histone deacetylase inhibitors and anticancer therapy. Curr Med Chem Anti-Cancer Agents 2: 477-484, 2002.

8. Rosato RR and Grant S: Histone deacetylase inhibitors in cancer therapy. Cancer Biol Ther 2: 30-37, 2003.

9. Kosugi H, Towatari M, Hatano S, Kitamura K, Kiyoi H, Kinoshita T, Tanimoto M, Murate T, Kawashima K, Saito H and Naoe T: Histone deacetylase inhibitors are the potent inducer/enhancer of differentiation in acute myeloid leukemia: a new approach to anti-leukemia therapy. Leukemia 13: 1316-1324, 1999.

10. Hauge A, Manning AM, Hanlon KA, Huschtscha LI, Hart D and Paraskeva C: Sodium butyrate induces apoptosis in human colonic tumour cell lines in a p53-independent pathway: implications for the possible role of dietary fibre in the prevention of large-bowel cancer. Int J Cancer 55: 498-505, 1993.
11. Heerdt BG, Houston MA and Augenlicht LH: Potentiation by specific short-chain fatty acids of differentiation and apoptosis in human colonic carcinoma cell lines. Cancer Res 54: 3288-3293, 1994.

12. Singh B, Halestrap AP and Paraskeva C: Butyrate can act as a stimulator of growth or inducer of apoptosis in human colonic epithelial cell lines depending on the presence of alternative energy sources. Carcinogenesis 18: 1265-1270, 1997.

13. Barnard JA and Warwick G: Butyrate rapidly induces growth inhibition and differentiation in HT-29 cells. Cell Growth Differ 4: 495-501, 1993

14. Chen JS, Faller DV and Spanjaard RA: Short-chain fatty acid inhibitors of histone deacetylases: promising anticancer therapeutics? Curr Cancer Drug Targets 3: 219-236, 2003.

15. Patnaik A, Rowinsky EK, Villalona MA, Hammond LA, Britten CD, Siu LL, Goetz A, Felton SA, Burton S, Valone FH and Eckhardt SG: A phase I study of pivaloyloxymethyl butyrate, a prodrug of the differentiating agent butyric acid, in patients with advanced solid malignancies. Clin Cancer Res 8: 2142-2148, 2002.

16. Douillard JY, Bennouna J, Vavasseur F, Deporte-Fety R, Thomare P, Giacalone F and Meflah K: Phase I trial of interleukin-2 and high-dose arginine butyrate in metastatic colorectal cancer. Cancer Immunol Immunother 49: 56-61, 2000.

17. Ema M, Hirota K, Mimura J, Abe H, Yodoi J, Sogawa K, Poellinger L and Fujii-Kuriyama Y: Molecular mechanisms of transcription activation by HLF and HIFlalpha in response to hypoxia: their stabilization and redox signal-induced interaction with CBP/p300. EMBO J 18: 1905-1914, 1999.

18. Kim MS, Kwon HJ, Lee YM, Baek JH, Jang JE, Lee SW, Moon EJ, Kim HS, Lee SK, Chung HY, Kim CW and Kim KW: Histone deacetylases induce angiogenesis by negative regulation of tumor suppressor genes. Nat Med 7: 437-443, 2001.

19. Saiki I, Fujii H, Yoneda J, Abe F, Nakajima M, Tsuruo T and Azuma I: Role of aminopeptidase N (CD13) in tumor-cell invasion and extracellular matrix degradation. Int J Cancer 54: 137-143, 1993.

20. Phillips PG, Birnby LM and Narendran A: Hypoxia induces capillary network formation in cultured bovine pulmonary microvessel endothelial cells. Am J Physiol 268: L789-L800, 1995.

21. Tang N, Wang L, Esko J, Giordano FJ, Huang Y, Gerber HP, Ferrara $\mathrm{N}$ and Johnson RS: Loss of HIF-1alpha in endothelial cells disrupts a hypoxia-driven VEGF autocrine loop necessary for tumorigenesis. Cancer Cell 6: 485-495, 2004.

22. Qian DZ, Wang X, Kachhap SK, et al: The histone deacetylase inhibitor NVP-LAQ824 inhibits angiogenesis and has a greater antitumor effect in combination with the vascular endothelial growth factor receptor tyrosine kinase inhibitor PTK787/ ZK222584. Cancer Res 64: 6626-6634, 2004.

23. Lee YM, Kim SH, Kim HS, Son MJ, Nakajima H, Kwon HJ and Kim KW: Inhibition of hypoxia-induced angiogenesis by FK228, a specific histone deacetylase inhibitor, via suppression of HIF-1 $\alpha$ activity. Biochem Biophys Res Commun 300: 241-246, 2003.

24. Kong X, Lin Z, Liang D, Fath D, Sang N and Caro J: Histone deacetylase inhibitors induce VHL and ubiquitin-independent proteasomal degradation of hypoxia-inducible factor $1 \alpha$. Mol Cell Biol 26: 2019-2028, 2006

25. Yoo YG, Kong G and Lee MO: Metastasis-associated protein 1 enhances stability of hypoxia-inducible factor- $1 \alpha$ protein by recruiting histone deacetylase 1. EMBO J 25: 1231-1241, 2006. 